\title{
Robust Semi-Global Coordinated Tracking of Linear Multi-agent Systems with Input
}

\section{Saturation}

\author{
Housheng Su, Michael Z. Q. Chen, and Guanrong Chen,
}

\begin{abstract}
This paper investigates the problem of coordinated tracking of a linear multi-agent system subject to actuator magnitude saturation and dead zone characteristic with input additive uncertainties and disturbances. Distributed consensus and swarm tracking protocols are developed from a low-and-high gain feedback approach. Under the assumption that each agent is asymptotically null controllable with bounded controls, it is shown that robust semi-global consensus tracking and swarm tracking of the multiagent system can always be reached provided that the networks are connected. Numerical examples are provided to illustrate the theoretical results.
\end{abstract}

\section{Index Terms}

Coordinated tracking, consensus, swarm, input saturation, low-and-high gain feedback.

$\mathrm{H}$. Su is with the School of Automation, Image Processing and Intelligent Control Key Laboratory of Education Ministry of China, Huazhong University of Science and Technology, Luoyu Road 1037, Wuhan 430074, China. Email: houshengsu@gmail.com.

M. Z. Q. Chen is with the Department of Mechanical Engineering, The University of Hong Kong, Hong Kong. Email: mzqchen@hku.hk.

G. Chen is with the Department of Electronic Engineering, City University of Hong Kong, Hong Kong, China. Email: eegchen@cityu.edu.hk.

This work was supported in part by the National Natural Science Foundation of China under Grant Nos. 61104140 and 61374053, the Program for New Century Excellent Talents in University from Chinese Ministry of Education under Grant NCET-12-0215, the Program for Changjiang Scholars and Innovative Research Team in University under Grant IRT1245, HKU CRCG 201111159110, and “973 Program” 2012CB720200. 


\section{INTRODUCTION}

Recently, multi-agent systems have been widely studied in different fields, including physics, biology, computer science, and control engineering, which accelerates the development of systems science and technology [1], [2]. In particular, research on distributed cooperative control protocols has advanced the development of cooperative control in multi-agent systems [3], [4], [5], [6], [7], [8], [9], [10], [11], [12]. For practical applications, various cooperative control capabilities need to be developed, including coordinated control without leaders and coordinated tracking to a leader in a multi-agent system.

Coordinated tracking of a multi-agent system means that a large number of followers, each relying only on its neighbors' local information, reach a coordinated motion with the predesigned leader, which are characterized by distributed control, local interactions and self-organization. Typical coordinated tracking of multi-agent systems includes consensus tracking and swarm tracking [13]. In previous studies, the coordinated tracking problem was first discussed for singleintegrator kinematics [14], [15], [16], and then generalized to double-integrator dynamics [17], [18], [19], [20], [21], [22] and general linear or nonlinear dynamics [23], [24], [25], [26], [27], [28], [29], [30].

This work considers (possibly time-varying) disturbance uncertainties and saturation constraints. These issues are not only theoretically challenging but also practically important. In physical and engineering systems, saturation nonlinearities are omnipresent because of the energy constraints and input signals saturation. Thus far, only a few studies have taken the saturation constraints into consideration [20], [31], [32], [33], [34], [35], [36], [37], [38], for example, to solve the consensus problem in multi-agent systems with single-integrator kinematics [31], [32], [33] or double-integrator dynamics [20], [34], [35]. In [36], a distributed projected consensus algorithm is developed for consensus problems. Without using velocity measurement, the consensus of multi-agent systems with double-integrator dynamics is investigated with input saturation in [37]. Under the assumptions that each agent is asymptotically null controllable with bounded controls and that the network is connected or jointly connected, low gain feedback based distributed consensus protocols are developed to achieve semi-global leader-following consensus of the multi-agent system without additive input uncertainties and disturbances [38].

In this paper, we address the coordinated tracking problem for a linear multi-agent system 
subject to actuator magnitude saturation and the dead zone constraint with additive input uncertainties and disturbances. In order to stabilize a single control system subject to actuator magnitude saturation and the dead zone characteristic as well as input additive uncertainties and disturbances, the control system needs to be asymptotically null controllable with bounded controls. Therefore, in this paper, we also consider the case that the agents are asymptotically null controllable with bounded controls. By utilizing the low-and-high gain design technique [39], we design coordinated tracking algorithms that can achieve robust semi-global consensus tracking or swarm tracking of multi-agent systems on a fixed communication network. Comparing to the existing works on similar topics, the contributions of this paper are three-fold. First, to the best of our knowledge, no existing work considered multi-agent systems subject to actuator magnitude saturation and the dead zone characteristic as well as input additive uncertainties and disturbances. Under such a general setting, this work is much closer to the practical situations. Second, this work extends the existing results on consensus with saturation constraints [20], [31], [32], [33], [34], [35], [36], [37] to the case of agents described by more general linear dynamics. Finally, differing from the low gain feedback consensus of linear multi-agent systems in [38], the low-and-high gain feedback strategy is adopted in this paper, which is more complex than the low gain feedback one. More precisely, we construct a family of low-and-high gain feedback laws, where the low gain controller is parameterized by a constant $\varepsilon>0$, which is completely separated from the high gain parameter $\rho>0$. For any a priori given bounded set in the state space, the value of $\varepsilon$ can be tuned to be small enough so as to increase the size of the equilibrium region of the closed-loop system in order to achieve coordinated tracking, as long as the network is connected and the agents are asymptotically null controllable with bounded controls. On the other hand, the high gain parameter plays a key role in achieving desirable performances beyond stabilization such as disturbance rejection, robustness and enhancement of control abilities.

The rest of the paper is organized as follows. Section II formulates the semi-global coordinated tracking problem to be studied in this paper. Section III presents the main results on the robust coordinated tracking problem. Section IV illustrates the theoretical results with numerical examples. Section V draws conclusions to the paper. 


\section{Problem Statement}

Consider a group of $N$ agents with general linear dynamics, labeled as $1,2, \ldots, N$, described by

$$
\dot{x}_{i}=A x_{i}+B u_{i}, \quad i=1,2, \ldots, N
$$

where $x_{i} \in \mathbf{R}^{n}$ is the state of agent $i$, and $u_{i} \in \mathbf{R}^{m}$ is the control input acting on agent $i ; A \in$ $\mathbf{R}^{n \times n}$ and $B \in \mathbf{R}^{n \times m}$ are known constant matrices. In the following, let $x=\left[x_{1}{ }^{\mathrm{T}}, x_{2}{ }^{\mathrm{T}}, \ldots, x_{N}{ }^{\mathrm{T}}\right]^{\mathrm{T}}$ and $u=\left[u_{1}{ }^{\mathrm{T}}, u_{2}{ }^{\mathrm{T}}, \ldots, u_{N}{ }^{\mathrm{T}}\right]^{\mathrm{T}}$. The dynamics of the leader, labeled as $N+1$, is described by

$$
\dot{x}_{N+1}=A x_{N+1} .
$$

The problem of robust semi-global consensus tracking for the agents and with a leader as described above is the following: For any given bounded set $\mathcal{X} \subset \mathbf{R}^{n}$, construct a control law $u_{i}$ for each agent $i$, which is affected by (possibly time-varying) disturbance uncertainties and only uses local information from neighbor agents, such that

$$
\lim _{t \rightarrow \infty}\left\|x_{i}(t)-x_{N+1}(t)\right\|=0, \quad i=1,2, \ldots, N .
$$

The problem of robust semi-global swarm tracking is similarly defined if the state difference between each agent and the leader, $\left\|x_{i}(t)-x_{N+1}(t)\right\|, i=1,2, \ldots, N$, remains in a prescribed set $\mathcal{X}_{0} \subset \mathbf{R}^{n}$ with $0 \in \mathcal{X}_{0}$, after some finite time, provided that $x_{i}(0) \in \mathcal{X}$ for all $i=1,2, \ldots, N, N+$ 1.

We consider the problem of robust semi-global coordinated tracking when the communication network is fixed. The network consisting of $N$ agents is described by an undirected graph $G=\{V, E\}$. In this graph, the set of vertices $V=\{1,2, \ldots, N\}$ represents the agents in the group and the set of edges $E=\{(i, j) \in V \times V: i \sim j\}$, containing unordered pairs of vertices, represents neighboring relations among the agents. Vertices $i$ and $j$ are said to be adjacent if $(i, j) \in E$. The adjacency matrix $A=\left(a_{i j}\right)$ of graph $G$ is defined by letting $a_{i j}=1$ if $(i, j) \in E$, and $a_{i j}=0$ otherwise. The Laplacian of graph $G$ with adjacency matrix $A$ is given by $L=\Delta(A)-A$, where the degree matrix $\Delta(A)$ is a diagonal matrix with the $i$ th diagonal element given by $\sum_{j=1, j \neq i}^{N} a_{i j}$. Denote the eigenvalues of $L$ as $\lambda_{1}(L) \leq \lambda_{2}(L) \leq \cdots \leq \lambda_{N}(L)$. Then, $\lambda_{1}(L)=0$, which has a corresponding eigenvector $\mathbf{1}=\left[\begin{array}{llll}1 & 1 & \cdots & 1\end{array}\right]^{\mathrm{T}} \in \mathbf{R}^{N}$. Moreover, $G$ is connected if and only if $\lambda_{2}(L)>0$ [40]. The $n$-dimensional graph Laplacian is defined as $\widehat{L}=L \otimes I_{n}$, where $I_{n}$ is the identity matrix of order $n$ and $\otimes$ stands for the Kronecker 
product. Let $\bar{G}$ be a graph generated by graph $G$ and a leader (vertex $N+1$ ), and $L$ be the symmetric Laplacian of the undirected graph $G$ consisting of $N$ agents. If agent $i$ is a neighbor of the leader at time $t$, then denote it by $h_{i}=1$; otherwise, $h_{i}=0$. Finally, define matrix $H=\operatorname{diag}\left\{h_{1}, h_{2}, \ldots, h_{N}\right\}$.

Lemma 1: [14] Let $L$ be the Laplacian of an undirected graph $G$ consisting of $N$ agents. Let $\bar{G}$ be the graph consisting of the $N$ agents and a leader (vertex $N+1$ ), which contains a spanning tree with the leader as the root vertex. Then, $L+H>0$.

Lemma 2: [28] Let $L_{1}$ and $L_{2}$ be the symmetric Laplacians of graphs $G_{1}$ and $G_{2}$, respectively, both consisting of $N$ agents. Let $\bar{G}_{1}$ be a graph consisting of the $N$ agents and a leader (agent $N+1$ ). Assume that $\bar{G}_{1}$ contains a spanning tree. Let $\bar{G}_{2}$ be a graph generated by adding some edge(s) among the $N$ agents into the graph $\bar{G}_{1}$. Then, $\lambda_{i}\left(L_{2}+H\right) \geq \lambda_{i}\left(L_{1}+H\right)>0$, $i=1,2, \cdots, N$.

Remark 1: Let $\bar{G}_{s}$ be a spanning tree consisting of the $N$ agents and a leader (agent $N+1$ ), and $\bar{G}_{s 1}$ be a graph generated by adding some edge(s) among the $N$ agents into the graph $\bar{G}_{s}$. Let $L_{s}$ and $L_{s 1}$ be, respectively, the corresponding symmetric Laplacians of $G_{s}$ and $G_{s 1}$, both consisting of the $N$ agents. Then, $\lambda_{1}\left(L_{s}+H\right) \leq \lambda_{1}\left(L_{s 1}+H\right)$. Since the number of the vertices of the spanning tree is finite and fixed, the number of possible spanning trees consisting of the $N$ agents and the leader is finite. Therefore, one can obtain the minimum value of $\lambda_{1}\left(L_{s}+H\right)$, denoted $\min \left\{\lambda_{1}\left(L_{s}+H\right)\right\}$. Let $\bar{G}_{c}$ be a complete graph consisting of the $N$ agents and the leader and $L_{c}$ be the corresponding symmetric Laplacian of $G_{c}$ consisting of the $N$ agents. By using a similar argument, one can show that $\lambda_{N}\left(L_{c}+H\right)$ is the maximum value of $\lambda_{N}(L+H)$.

\section{MAIn RESULTS}

To find a solution to the problem of robust semi-global coordinated tracking, the following assumptions are needed.

Assumption 1: The pair $(A, B)$ is asymptotically null controllable with bounded controls, that is, $(A, B)$ is stabilizable and all the eigenvalues of $A$ are in the closed left-half plane.

Assumption 2: The graph $\bar{G}$ consisting of the $N$ agents and the leader contains a spanning tree rooted at the leader.

Lemma 3: [39] Let Assumption 1 hold. Then, for each $\varepsilon \in(0,1]$, there exists a unique matrix 
$P(\varepsilon)>0$ that solves the algebraic Riccati equation (ARE)

$$
A^{\mathrm{T}} P(\varepsilon)+P(\varepsilon) A-P(\varepsilon) B B^{\mathrm{T}} P(\varepsilon)+\varepsilon I=0 .
$$

Moreover, $\lim _{\varepsilon \rightarrow 0} P(\varepsilon)=0$.

Definition 1: A saturation function $\sigma: \mathbf{R}^{m} \rightarrow \mathbf{R}^{m}$ satisfies

(1) $\sigma(s)$ is decentralized, that is, $\sigma(s)=\left[\sigma_{1}\left(s_{1}\right) \sigma_{2}\left(s_{2}\right) \cdots \sigma_{m}\left(s_{m}\right)\right]^{\mathrm{T}}, s=\left[s_{1}, s_{2}, \ldots, s_{m}\right]^{\mathrm{T}} \in$ $\mathbf{R}^{m} ;$ and

(2) for each $i=1,2, \ldots, m$,

$$
\sigma_{i}\left(s_{i}\right)=\left\{\begin{array}{lll}
0, & \text { if } & \left|s_{i}\right|<b, \\
s_{i}-\operatorname{sign}\left(s_{i}\right) b, & \text { if } & b \leq\left|s_{i}\right| \leq b+\delta, \\
\delta, & \text { if } & s_{i}>b+\delta, \\
-\delta, & \text { if } & s_{i}<-b-\delta,
\end{array}\right.
$$

where $\operatorname{sign}(\cdot)$ is the signum function, and the constants $\delta>0$ and $b \geq 0$.

Note that if $b=0$, the saturation function $\sigma(\cdot)$ is the standard saturation function, and if $b>0$, the saturation function $\sigma(\cdot)$ is the standard saturation function with ideal dead zone characteristics.

The proposed low-and-high gain feedback design for the multi-agent system (1) is carried out in two steps.

Low-and-high gain-based coordinated tracking control protocol:

Step 1. Solve the parametric algebraic Riccati equation (ARE)

$$
A^{\mathrm{T}} P(\varepsilon)+P(\varepsilon) A-2 \gamma P(\varepsilon) B B^{\mathrm{T}} P(\varepsilon)+\varepsilon I=0, \quad \varepsilon \in(0,1],
$$

where $\gamma \leq \min \left\{\lambda_{1}\left(L_{s}+H\right)\right\}$ is a positive constant. The existence of a unique positive definite solution $P(\varepsilon)$ for the ARE (3) is guaranteed by Lemma 3.

Step 2. Construct a feedback law for agent $i$ as

$$
\begin{aligned}
u_{i}= & -\sum_{j=1}^{N} a_{i j} \sigma\left((1+\rho) B^{\mathrm{T}} P(\varepsilon)\left(x_{i}-x_{j}\right)+g\left(x_{i}-x_{j}, t\right)\right) \\
& -h_{i} \sigma\left((1+\rho) B^{\mathrm{T}} P(\varepsilon)\left(x_{i}-x_{N+1}\right)+g\left(x_{i}-x_{N+1}, t\right)\right), \quad i=1,2, \ldots, N,
\end{aligned}
$$

where the nonnegative parameters $\rho$ and $\varepsilon$ are referred to as the high gain parameter and the low gain parameter, respectively. The function $g: \mathbf{R}^{n} \times \mathbf{R}_{+} \rightarrow \mathbf{R}^{m}$ represents (possibly time-varying) disturbance uncertainties. 
Remark 2: In the above design algorithm, the control inputs are bounded and each follower agent only acquires the state information of its neighbors. Moreover, the design algorithm requires no information of the network topology. If the number of agents, $N$, is known, the value of $\gamma$ is determined.

Assumption 3: [39] The uncertain element

$$
g(v, t)=\left[g_{1}(v, t), g_{2}(v, t), \cdots, g_{m}(v, t)\right]=-g(-v, t) \in \mathbf{R}^{m}
$$

is piecewise continuous in $t$, locally Lipschitz in $v$ and its norm is bounded by a known function

$$
|g(v, t)| \leq g_{0}(|v|)+D_{0}, \forall(v, t) \in \mathbf{R}^{n} \times \mathbf{R}_{+},
$$

where $D_{0}$ is a known nonnegative constant, and the known function $g_{0}: \mathbf{R}_{+} \rightarrow \mathbf{R}_{+}$is locally Lipschitz and satisfies $g_{0}(0)=0$.

Lemma 4: For any $K \in \mathbf{R}^{m \times n}$ and any $\varsigma_{i}, \xi_{i} \in \mathbf{R}^{m}, i=1,2, \ldots, N$,

$$
\begin{aligned}
& \frac{1}{2} \sum_{i=1}^{N} \sum_{j=1}^{N} a_{i j}\left(\varsigma_{i}-\varsigma_{j}\right)^{\mathrm{T}} \sigma\left(K\left(\xi_{i}-\xi_{j}\right)+g\left(\xi_{i}-\xi_{j}, t\right)\right) \\
= & \sum_{i=1}^{N} \sum_{j=1}^{N} a_{i j} \varsigma_{i}^{\mathrm{T}} \sigma\left(K\left(\xi_{i}-\xi_{j}\right)+g\left(\xi_{i}-\xi_{j}, t\right)\right) .
\end{aligned}
$$

Proof. The proof involves a simple rearrangement of terms in the following double summation

$$
\begin{aligned}
& \frac{1}{2} \sum_{i=1}^{N} \sum_{j=1}^{N} a_{i j}\left(\varsigma_{i}-\varsigma_{j}\right)^{\mathrm{T}} \sigma\left(K\left(\xi_{i}-\xi_{j}\right)+g\left(\xi_{i}-\xi_{j}, t\right)\right) \\
= & \frac{1}{2} \sum_{i=1}^{N} \sum_{j=1}^{N} a_{i j} \varsigma_{i}^{\mathrm{T}} \sigma\left(K\left(\xi_{i}-\xi_{j}\right)+g\left(\xi_{i}-\xi_{j}, t\right)\right) \\
& -\frac{1}{2} \sum_{i=1}^{N} \sum_{j=1}^{N} a_{i j} \varsigma_{j}^{\mathrm{T}} \sigma\left(K\left(\xi_{i}-\xi_{j}\right)+g\left(\xi_{i}-\xi_{j}, t\right)\right) \\
= & \frac{1}{2} \sum_{i=1}^{N} \sum_{j=1}^{N} a_{i j} \varsigma_{i}^{\mathrm{T}} \sigma\left(K\left(\xi_{i}-\xi_{j}\right)+g\left(\xi_{i}-\xi_{j}, t\right)\right) \\
& +\frac{1}{2} \sum_{j=1}^{N} \sum_{i=1}^{N} a_{j i} \varsigma_{i}^{\mathrm{T}} \sigma\left(K\left(\xi_{i}-\xi_{j}\right)+g\left(\xi_{i}-\xi_{j}, t\right)\right) \\
= & \sum_{i=1}^{N} \sum_{j=1}^{N} a_{i j} \varsigma_{i}^{\mathrm{T}} \sigma\left(K\left(\xi_{i}-\xi_{j}\right)+g\left(\xi_{i}-\xi_{j}, t\right)\right) .
\end{aligned}
$$

This complete the proof. 
Lemma 5: Let $K \in \mathbf{R}^{m \times n}$ and $z \in \mathbf{R}^{n}, w=K z=\left[w_{1}, w_{2}, \cdots, w_{m}\right]^{\mathrm{T}} \in \mathbf{R}^{m}$, and $0<|z| \leq$ $z_{\max }$. If $\left|\rho w_{i}\right| \geq\left|g_{i}(z, t)\right|+b$,

$$
-w_{i}\left[\sigma_{i}\left((1+\rho) w_{i}+g_{i}(z, t)\right)-\operatorname{sat}_{\delta}\left(w_{i}\right)\right] \leq 0
$$

where $\operatorname{sat}_{\delta}(\cdot)$ is a standard saturation function. If $\left|\rho w_{i}\right| \leq\left|g_{i}(z, t)\right|+b$,

$$
-w_{i}\left[\sigma_{i}\left((1+\rho) w_{i}+g_{i}(z, t)\right)-\operatorname{sat}_{\delta}\left(w_{i}\right)\right] \leq \frac{6}{\rho}\left(D_{0}^{2}+b^{2}+M^{2}|z|^{2}\right),
$$

where $M=\sup _{d \in\left(0, z_{\max }\right]}\left\{\frac{g_{0}(d)}{d}\right\}$.

Proof. It follows from Definition 1 that, for each $i$,

$$
s_{i}\left[\sigma_{i}\left(s_{i}+\operatorname{sign}\left(s_{i}\right) \bar{b}\right)-\operatorname{sat}_{\delta}\left(s_{i}\right)\right] \geq 0, \forall s_{i} \in \mathbf{R}, \bar{b} \geq b .
$$

Therefore, if $\left|\rho w_{i}\right| \geq\left|g_{i}(z, t)\right|+b$,

$$
-w_{i}\left[\sigma_{i}\left((1+\rho) w_{i}+g_{i}(z, t)\right)-\operatorname{sat}_{\delta}\left(w_{i}\right)\right] \leq 0
$$

Otherwise, if $\left|\rho w_{i}\right| \leq\left|g_{i}(z, t)\right|+b$,

$$
\begin{aligned}
& -w_{i}\left[\sigma_{i}\left((1+\rho) w_{i}+g_{i}(z, t)\right)-\operatorname{sat}_{\delta}\left(w_{i}\right)\right] \\
= & -w_{i}\left[\sigma_{i}\left((1+\rho) w_{i}+g_{i}(z, t)\right)-\sigma_{i}\left(w_{i}\right)\right] \\
& -w_{i}\left[\sigma_{i}\left(w_{i}\right)-\sigma_{i}\left(w_{i}+\operatorname{sign}\left(w_{i}\right) b\right)\right] \\
& -w_{i}\left[\sigma_{i}\left(w_{i}+\operatorname{sign}\left(w_{i}\right) b\right)-\operatorname{sat}_{\delta}\left(w_{i}\right)\right] \\
\leq & \frac{\left(\left|g_{i}(z, t)\right|+b\right)}{\rho}\left(2\left|g_{i}(z, t)\right|+b+b\right) \\
\leq & \frac{2}{\rho}\left(g_{0}(|z|)+b+D_{0}\right)^{2} \\
\leq & \frac{6}{\rho}\left(D_{0}^{2}+b^{2}+M^{2}|z|^{2}\right) .
\end{aligned}
$$

This completes the proof.

Let

$$
\begin{gathered}
\bar{M}=\sup _{d \in(0, F]}\left\{\frac{g_{0}(d)}{d}\right\}, \\
F=\sqrt{c \lambda_{\min }^{-1}(P(\varepsilon))}, \\
\theta=\lambda_{N}\left(L_{c}+H\right),
\end{gathered}
$$




$$
\begin{gathered}
\rho_{1}^{*}=12 m \bar{M}^{2} \frac{\theta \lambda_{\max }(P(\varepsilon))}{\varepsilon \lambda_{\min }(P(\varepsilon))}, \\
\rho_{2}^{*}=N(N-1) \frac{24 m \lambda_{\max }(P(\varepsilon))}{\varepsilon c}\left(D_{0}^{2}+b^{2}\right),
\end{gathered}
$$

and

$$
\rho \geq \max \left\{\rho_{1}^{*}, \rho_{2}^{*}\right\}
$$

The following is the main results on the robust semi-global coordinated tracking problem of the multi-agent system.

Theorem 1: Consider a multi-agent system of $N$ agents described by general linear dynamics (1), with a leader described by dynamics (2). Suppose that Assumptions 1, 2 and 3 hold. Then, the control inputs $u_{i}$ for the agent (4) will achieve robust semi-global consensus tracking of the multi-agent system if $D_{0}=0$ and $b=0$; or achieve robust semi-global swarm tracking of the multi-agent system if $D_{0}>0$ or $b>0$. That is, for any given bounded set $\mathcal{X} \subset \mathbf{R}^{n}$, there is an $\varepsilon^{*}(\delta, \mathcal{X})>0$ and, for each $\varepsilon \in\left(0, \varepsilon^{*}\right]$, there exists a $\rho^{*}\left(\varepsilon, \delta, b, g_{0}, D_{0}, \mathcal{X}, \mathcal{X}_{0}\right)$ such that, for any given $\varepsilon \in\left(0, \varepsilon^{*}\right]$ and $\rho \geq \rho^{*}$, if $D_{0}=0$ and $b=0$,

$$
\lim _{t \rightarrow \infty}\left\|x_{i}(t)-x_{N+1}(t)\right\|=0, \quad i=1,2, \ldots, N
$$

provided that $x_{i}(0) \in \mathcal{X}$ for all $i=1,2, \ldots, N, N+1$; if $D_{0}>0$ or $b>0$, the state difference between the agent and the leader, $\left\|x_{i}(t)-x_{N+1}(t)\right\|, i=1,2, \ldots, N$, remains in a set $\mathcal{X}_{0} \subset \mathbf{R}^{n}$ with $0 \in \mathcal{X}_{0}$ after some finite time, provided that $x_{i}(0) \in \mathcal{X}$ for all $i=1,2, \ldots, N, N+1$.

Proof. Part i). In this part, we firstly use the difference between the states of agents and the leader to construct an error system, which is equivalent to the original system. Base on the error system, we define a Lyapunov function and a set, and calculate the derivative of the Lyapunov function along the trajectories of the agents within the set.

Denote the difference between the states of agent $i$ and the leader as $\tilde{x}_{i}=x_{i}-x_{N+1}, i=$ $1,2, \ldots, N$. Then,

$$
\begin{aligned}
\dot{\tilde{x}}_{i}= & A \tilde{x}_{i}+B u_{i} \\
= & A \tilde{x}_{i}-B \sum_{j=1}^{N} a_{i j} \sigma\left((1+\rho) B^{\mathrm{T}} P(\varepsilon)\left(\tilde{x}_{i}-\tilde{x}_{j}\right)+g\left(\tilde{x}_{i}-\tilde{x}_{j}, t\right)\right) \\
& -B h_{i} \sigma\left((1+\rho) B^{\mathrm{T}} P(\varepsilon) \tilde{x}_{i}+g\left(\tilde{x}_{i}, t\right)\right) .
\end{aligned}
$$


Consider the Lyapunov function

$$
V(\tilde{x})=\sum_{i=1}^{N} \tilde{x}_{i}^{\mathrm{T}} P(\varepsilon) \tilde{x}_{i}
$$

where $\tilde{x}=\left[\begin{array}{llll}\tilde{x}_{1}^{\mathrm{T}} & x_{2}^{\mathrm{T}} & \cdots & \tilde{x}_{N}^{\mathrm{T}}\end{array}\right]^{\mathrm{T}}$. Let $c>0$ be a constant such that

$$
c \geq \sup _{\varepsilon \in(0,1], x_{i}(0) \in \mathcal{X}, i=1,2, \ldots, N+1} \sum_{i=1}^{N} \tilde{x}_{i}^{\mathrm{T}}(0) P(\varepsilon) \tilde{x}_{i}(0) .
$$

Such a $c$ exists since $\mathcal{X}$ is bounded and $\lim _{\varepsilon \rightarrow 0} P(\varepsilon)=0$ by Lemma 3 .

Let $L_{V}(c):=\left\{\tilde{x} \in \mathbf{R}^{N n}: V(\tilde{x}) \leq c\right\}$, and let $\varepsilon^{*} \in(0,1]$ be such that, for each $\varepsilon \in\left(0, \varepsilon^{*}\right]$, $\tilde{x} \in L_{V}(c)$ implies that

$$
\begin{aligned}
\left\|B^{\mathrm{T}} P(\varepsilon) \tilde{x}_{i}\right\|_{\infty} & \leq \delta \\
\left\|B^{\mathrm{T}} P(\varepsilon)\left(\tilde{x}_{i}-\tilde{x}_{j}\right)\right\|_{\infty} & \leq \delta, \quad i=1,2, \ldots, N
\end{aligned}
$$

where $\|z\|_{\infty}=\max _{i}\left|z_{i}\right|$ for $z \in \mathbf{R}^{n}$. The existence of such an $\varepsilon^{*}$ is again due to the fact that $\lim _{\varepsilon \rightarrow 0} P(\varepsilon)=0$. Thus, the derivative of $V$ along the trajectories of the agents within the set $L_{V}(c)$ is given by

$$
\begin{aligned}
\dot{V}(\tilde{x})= & \dot{x}^{\mathrm{T}} P(\varepsilon) \tilde{x}+\tilde{x}^{\mathrm{T}} P(\varepsilon) \dot{\tilde{x}} \\
= & \sum_{i=1}^{N} \tilde{x}_{i}^{\mathrm{T}}\left(P(\varepsilon) A+A^{\mathrm{T}} P(\varepsilon)\right) \tilde{x}_{i} \\
& -2 \sum_{i=1}^{N} \tilde{x}_{i}^{\mathrm{T}} P(\varepsilon) B \sum_{j=1}^{N} a_{i j} \sigma\left((1+\rho) B^{\mathrm{T}} P(\varepsilon)\left(\tilde{x}_{i}-\tilde{x}_{j}\right)+g\left(\tilde{x}_{i}-\tilde{x}_{j}, t\right)\right) \\
& -2 \sum_{i=1}^{N} \tilde{x}_{i}^{\mathrm{T}} P(\varepsilon) B h_{i} \sigma\left((1+\rho) B^{\mathrm{T}} P(\varepsilon) \tilde{x}_{i}+g\left(\tilde{x}_{i}, t\right)\right) \\
= & \sum_{i=1}^{N} \tilde{x}_{i}^{\mathrm{T}}\left(P(\varepsilon) A+A^{\mathrm{T}} P(\varepsilon)\right) \tilde{x}_{i} \\
& -2 \sum_{i=1}^{N} \tilde{x}_{i}^{\mathrm{T}} P(\varepsilon) B B^{\mathrm{T}} P(\varepsilon)\left(\sum_{j=1}^{N} a_{i j}\left(\tilde{x}_{i}-\tilde{x}_{j}\right)+h_{i} \tilde{x}_{i}\right) \\
& -\sum_{i=1}^{N}\left(\tilde{x}_{i}-\tilde{x}_{j}\right)^{\mathrm{T}} P(\varepsilon) B \sum_{j=1}^{N} a_{i j} \sigma\left((1+\rho) B^{\mathrm{T}} P(\varepsilon)\left(\tilde{x}_{i}-\tilde{x}_{j}\right)+g\left(\tilde{x}_{i}-\tilde{x}_{j}, t\right)\right) \\
& +\sum_{i=1}^{N}\left(\tilde{x}_{i}-\tilde{x}_{j}\right)^{\mathrm{T}} P(\varepsilon) B \sum_{j=1}^{N} a_{i j} B^{\mathrm{T}} P(\varepsilon)\left(\tilde{x}_{i}-\tilde{x}_{j}\right) \\
& -2 \sum_{i=1}^{N} \tilde{x}_{i}^{\mathrm{T}} P(\varepsilon) B h_{i}\left(\sigma\left((1+\rho) B^{\mathrm{T}} P(\varepsilon) \tilde{x}_{i}+g\left(\tilde{x}_{i}, t\right)\right)-B^{\mathrm{T}} P(\varepsilon) \tilde{x}_{i}\right) .
\end{aligned}
$$


Part ii). In this part, we consider the derivative of the Lyapunov function along the trajectories of the agents within the set in two cases. In the first case, the derivative of the Lyapunov function along the trajectories of the agents within the set is always negative. In the second case, the derivative of the Lyapunov function along the trajectories of the agents within the set is not always negative, but it is negative when the value of the Lyapunov function is close to the boundary value.

Now consider two cases, i) and ii), as follows.

i) If $\left|\rho B^{\mathrm{T}} P(\varepsilon)\left(\tilde{x}_{i}-\tilde{x}_{j}\right)\right| \geq\left|g_{i}\left(\tilde{x}_{i}-\tilde{x}_{j}, t\right)\right|+b$ and $\left|\rho B^{\mathrm{T}} P(\varepsilon) \tilde{x}_{i}\right| \geq\left|g_{i}\left(\tilde{x}_{i}, t\right)\right|+b$, for all $i=$ $1,2,, \cdots, N$, then from Lemma 5 and Equation (9), one has

$$
\begin{aligned}
\dot{V}(\tilde{x})= & \sum_{i=1}^{N} \tilde{x}_{i}^{\mathrm{T}}\left(P(\varepsilon) A+A^{\mathrm{T}} P(\varepsilon)\right) \tilde{x}_{i} \\
& -\sum_{i=1}^{N} \sum_{j=1}^{N} a_{i j}\left(\tilde{x}_{i}^{\mathrm{T}}-\tilde{x}_{j}^{\mathrm{T}}\right) P(\varepsilon) B B^{\mathrm{T}} P(\varepsilon)\left(\tilde{x}_{i}-\tilde{x}_{j}\right) \\
& -2 \sum_{i=1}^{N} h_{i} \tilde{x}_{i}^{\mathrm{T}} P(\varepsilon) B B^{\mathrm{T}} P(\varepsilon) \tilde{x}_{i} .
\end{aligned}
$$

Recalling from [21] the fact that for any $\xi_{i} \in \mathbf{R}^{m}, i=1,2, \ldots, N$,

$$
\frac{1}{2} \sum_{i=1}^{N} \sum_{j=1}^{N} a_{i j}\left(\xi_{i}-\xi_{j}\right)^{\mathrm{T}}\left(\xi_{i}-\xi_{j}\right)=\xi^{\mathrm{T}}\left(L \otimes I_{m}\right) \xi,
$$

where $\xi=\left[\begin{array}{llll}\xi_{1} & \xi_{2} & \cdots & \xi_{N}\end{array}\right]^{\mathrm{T}}$, and using the identity $(A \otimes B)(C \otimes D)=A C \otimes B D$, one can obtain from (10) that

$$
\begin{aligned}
\dot{V}(\tilde{x})= & \tilde{x}^{\mathrm{T}}\left(I_{N} \otimes\left(P(\varepsilon) A+A^{\mathrm{T}} P(\varepsilon)\right)\right) \tilde{x} \\
& -2 \tilde{x}^{\mathrm{T}}\left(I_{N} \otimes P(\varepsilon) B\right)\left(L \otimes I_{m}\right)\left(I_{N} \otimes B^{\mathrm{T}} P(\varepsilon)\right) \tilde{x} \\
& -2 \tilde{x}^{\mathrm{T}}\left(I_{N} \otimes P(\varepsilon) B\right)\left(H \otimes I_{m}\right)\left(I_{N} \otimes B^{\mathrm{T}} P(\varepsilon)\right) \tilde{x} \\
= & \tilde{x}^{\mathrm{T}}\left(I_{N} \otimes\left(P(\varepsilon) A+A^{\mathrm{T}} P(\varepsilon)\right)\right) \tilde{x} . \\
& -\tilde{x}^{\mathrm{T}}\left((L+H) \otimes\left(2 P(\varepsilon) B B^{\mathrm{T}} P(\varepsilon)\right)\right) \tilde{x} .
\end{aligned}
$$

The symmetry of the matrix $L+H$ implies that there exists an orthogonal matrix $T \in \mathbf{R}^{N \times N}$ such that

$$
\begin{aligned}
L+H & =T^{\mathrm{T}} \operatorname{diag}\left\{\lambda_{1}(L+H), \lambda_{2}(L+H), \ldots, \lambda_{N}(L+H)\right\} T \\
& :=T^{\mathrm{T}} \operatorname{diag}\left\{\lambda_{1}, \lambda_{2}, \ldots, \lambda_{N}\right\} T .
\end{aligned}
$$


Thus, (11) further gives

$$
\begin{aligned}
\dot{V}(\tilde{x}) & =\tilde{x}^{\mathrm{T}}\left(\left(T^{\mathrm{T}} T\right) \otimes\left(P(\varepsilon) A+A^{\mathrm{T}} P(\varepsilon)\right)\right) \tilde{x} \\
& -\tilde{x}^{\mathrm{T}}\left(T^{\mathrm{T}} \operatorname{diag}\left\{\lambda_{1}, \ldots, \lambda_{N}\right\} T \otimes\left(2 P(\varepsilon) B B^{\mathrm{T}} P(\varepsilon)\right)\right) \tilde{x} \\
& =\sum_{i=1}^{N} \tilde{x}_{i}^{\mathrm{T}}\left(T^{\mathrm{T}} \otimes I_{n}\right)\left(P(\varepsilon) A+A^{\mathrm{T}} P(\varepsilon)-2 \lambda_{i} P(\varepsilon) B B^{\mathrm{T}} P(\varepsilon)\right)\left(T \otimes I_{n}\right) \tilde{x}_{i} \\
& \leq \sum_{i=1}^{N} \tilde{x}_{i}^{\mathrm{T}}\left(T^{\mathrm{T}} \otimes I_{n}\right)\left(P(\varepsilon) A+A^{\mathrm{T}} P(\varepsilon)-2 \gamma P(\varepsilon) B B^{\mathrm{T}} P(\varepsilon)\right)\left(T \otimes I_{n}\right) \tilde{x}_{i} \\
& =-\varepsilon \sum_{i=1}^{N} \tilde{x}_{i}^{\mathrm{T}}\left(T^{\mathrm{T}} \otimes I_{n}\right)\left(T \otimes I_{n}\right) \tilde{x}_{i} \\
< & 0, \quad \forall \tilde{x}_{i} \in L_{V}(c) \backslash\{0\} .
\end{aligned}
$$

ii) If the condition in case i) is not satisfied, then from Lemma 5 and (9), one has

$$
\begin{aligned}
\dot{V}(\tilde{x}) \leq & -\varepsilon \sum_{i=1}^{N} \tilde{x}_{i}^{\mathrm{T}} \tilde{x}_{i}+\sum_{i=1}^{N} \sum_{j=1}^{N} a_{i j} \frac{6 m}{\rho}\left(D_{0}^{2}+b^{2}+\bar{M}^{2}\left(\tilde{x}_{i}-\tilde{x}_{j}\right)^{\mathrm{T}}\left(\tilde{x}_{i}-\tilde{x}_{j}\right)\right) \\
& +2 \sum_{i=1}^{N} \sum_{j=1}^{N} h_{i} \frac{6 m}{\rho}\left(D_{0}^{2}+b^{2}+\bar{M}^{2} \tilde{x}_{i}^{\mathrm{T}} \tilde{x}_{i}\right) \\
\leq & -\left[\frac{\varepsilon}{2} \lambda_{\max }^{-1}(P(\varepsilon))-\frac{6 m}{\rho} \bar{M}^{2} \frac{\theta}{\lambda_{\min }(P(\varepsilon))}\right] V(\tilde{x}) \\
& -\frac{\varepsilon}{2} \lambda_{\max }^{-1}(P(\varepsilon)) V(\tilde{x})+\sum_{i=1}^{N} \sum_{j=1}^{N}\left(a_{i j}+2 h_{i}\right) \frac{6 m}{\rho}\left(D_{0}^{2}+b^{2}\right) \\
\leq & -\frac{\varepsilon}{2} \lambda_{\max }^{-1}(P(\varepsilon))\left[V(\tilde{x})-\frac{\rho_{2}^{*} c}{2 \rho}\right]
\end{aligned}
$$

Therefore,

$$
\begin{array}{r}
\tilde{x} \in\left\{\tilde{x} \in \mathbf{R}^{N n}: \frac{\rho_{2}^{*} c}{2 \rho} \leq V(\tilde{x}) \leq c\right\} \Rightarrow \dot{V}(\tilde{x})<0, \\
\forall \tilde{x}_{i} \in L_{V}(c) \backslash\{0\} .
\end{array}
$$

Now, suppose that the state difference between an agent and the leader, $\left\|x_{i}(t)-x_{N+1}(t)\right\|$, $i=1,2, \ldots, N$, enters into the set $B=\left\{\tilde{x} \in \mathbf{R}^{N n}: V(\tilde{x}) \leq \frac{c}{2}\right\}$ at time $T$. Then, $V(T)=\frac{c}{2}$ and, from (12), one has

$$
T \leq \frac{2 \lambda_{\max }(P(\varepsilon))}{\varepsilon} \ln \left(\frac{V(0)-\frac{\rho_{2}^{*} c}{2 \rho}}{\frac{c}{2}-\frac{\rho_{2}^{*} c}{2 \rho}}\right) .
$$

Part iii). In this part, we illustrate that the consensus tracking will be achieved when $D_{0}=0$ and $b=0$, and the swarm tracking will be attained when $D_{0}>0$ or $b>0$. 
When $D_{0}=0$ and $b=0$, one has $\rho_{2}^{*}=0$. This implies that the trajectory of $\tilde{x}$ starting from the level set $L_{V}(c)$ will converge to the origin asymptotically as time goes to infinity, which in turn implies that

$$
\lim _{t \rightarrow \infty}\left\|x_{i}(t)-x_{N+1}(t)\right\|=0, \quad i=1,2, \ldots, N .
$$

If $D_{0}>0$ or $b>0$, then the condition discussed in case i) is not satisfied and moreover $\rho_{2}^{*} \neq 0$. The state difference between an agent and the leader, $\left\|x_{i}(t)-x_{N+1}(t)\right\|, i=1,2, \ldots, N$, remains in a set $\mathcal{X}_{0} \subset L_{V}(c)$ with $0 \in \mathcal{X}_{0}$, provided that $x_{i}(0) \in \mathcal{X}$, for all $i=1,2, \ldots, N, N+1$. This completes the proof.

Remark 3: In this paper, we adopt the ARE-based method to deal with the coordinated tracking problem. Therefore, the resulting feedback gain is indirectly dependent on the low gain parameter $\varepsilon$. For each different value of $\varepsilon$, the solution of a parameterized ARE is required. Therefore, one can use the following bisectioning method to compute the parameter $\varepsilon^{*}$ and $\rho^{*}$ without using any global information of the multi-agent systems. First, we choose an $\varepsilon^{*} \in(0,1]$. From Equation (3), one can obtain the corresponding $P\left(\varepsilon^{*}\right)$. Note that the calculation of $\gamma$ in Equation (3) only depends on the number of the agents $N$. For a given bounded set $\mathcal{X} \subset \mathbf{R}^{n}$, one can find the maximum value of $\|\mu\|_{\infty}$, where $\|\mu\|_{\infty}=\max _{i}|\mu|$ for $\mu \in \mathcal{X}$. Let the maximum value of $\|\mu\|_{\infty}$ be $a$, and $x_{i}(0) \in \mathcal{X}$, one has $\left\|x_{i}(0)\right\|_{\infty} \leq a$. Let $\mathbf{1}=[1,1, \ldots, 1]^{T}$. Since $\tilde{x}_{i}=x_{i}-x_{N+1}$, from Equation (6), one has

$$
V(\tilde{x}(0)) \leq 4 a^{2} \sum_{i=1}^{N} \mathbf{1}^{T} P\left(\varepsilon^{*}\right) \mathbf{1} .
$$

Therefore, from Equation (7), one can design

$$
c=4 a^{2} \sum_{i=1}^{N} \mathbf{1}^{T} P\left(\varepsilon^{*}\right) \mathbf{1} .
$$

From Equation (6) and $\dot{V} \leq 0$, one has

$$
\tilde{x}_{i}^{T} P\left(\varepsilon^{*}\right) \tilde{x}_{i} \leq c .
$$

Thus, one obtains the maximum values of $\left\|\tilde{x}_{i}\right\|_{\infty}, i=1,2, \ldots, N$. Let the maximum value of $\left\|\tilde{x}_{i}\right\|_{\infty}$ be $g$ and

$$
B^{\mathrm{T}} P\left(\varepsilon^{*}\right)=\kappa=\left(\kappa_{i j}\right)_{m \times n} \in \mathbf{R}^{m \times n}
$$

Let

$$
\bar{\kappa}=\left(\left|\kappa_{i j}\right|\right)_{m \times n} \in \mathbf{R}^{m \times n},
$$


one has

$$
\begin{aligned}
& \left\|B^{\mathrm{T}} P\left(\varepsilon^{*}\right) \tilde{x}_{i}\right\|_{\infty} \leq\|g \bar{\kappa} \mathbf{1}\|_{\infty}, \\
& \left\|B^{\mathrm{T}} P\left(\varepsilon^{*}\right)\left(\tilde{x}_{i}-\tilde{x}_{j}\right)\right\|_{\infty} \leq\|2 g \bar{\kappa} \mathbf{1}\|_{\infty}, \\
& i=1,2, \ldots, N \text {. }
\end{aligned}
$$

If $\|2 g \bar{\kappa} \mathbf{1}\|_{\infty} \leq \delta$, then the parameter $\varepsilon^{*}$ is feasible. Otherwise, one uses the half value of the former given value $\varepsilon^{*}$ to repeat the above calculation process until the saturation constraint $\|2 g \bar{\kappa} \mathbf{1}\|_{\infty} \leq \delta$ is satisfied. Therefore,

$$
\begin{gathered}
\bar{M}=\sup _{d \in(0, F]}\left\{\frac{g_{0}(d)}{d}\right\}, \\
F=\sqrt{c \lambda_{\min }^{-1}\left(P\left(\varepsilon^{*}\right)\right)}, \\
\theta=\lambda_{N}\left(L_{c}+H\right), \\
\rho_{1}^{*}=12 m \bar{M}^{2} \frac{\theta \lambda_{\max }\left(P\left(\varepsilon^{*}\right)\right)}{\varepsilon \lambda_{\min }\left(P\left(\varepsilon^{*}\right)\right)}, \\
\rho_{2}^{*}=N(N-1) \frac{24 m \lambda_{\max }\left(P\left(\varepsilon^{*}\right)\right)}{\varepsilon^{*} c}\left(D_{0}^{2}+b^{2}\right),
\end{gathered}
$$

and

$$
\rho^{*}=\max \left\{\rho_{1}^{*}, \rho_{2}^{*}\right\}
$$

\section{NUMERICAL EXAMPLES}

The simulation is performed with ten agents and one leader. The system matrices are chosen as

$$
A=\left[\begin{array}{cccc}
0 & 1 & 0 & 0 \\
0 & 0 & 1 & 0 \\
0 & 0 & 0 & 1 \\
-1 & 0 & -4 & 0
\end{array}\right], B=\left[\begin{array}{l}
0 \\
0 \\
0 \\
1
\end{array}\right]
$$

It is straightforward to verify that $(A, B)$ is asymptotically null controllable with bounded controls. The disturbance uncertainties function $g\left(x_{i}, t\right)=0.002 \sin t \cdot \sum_{j=1}^{n} x_{i j}$, the known function $g_{0}\left(\left|x_{i}\right|\right)=0.002\left|x_{i}\right|$ and the constant $\delta=3$. The initial states $x_{i 1}, x_{i 2}, x_{i 3} \ldots x_{i 10}$ of all agents are randomly chosen from the box $[-3,3] \times[-3,3] \times[-3,3] \times[-3,3]$, and the 
initial state of the leader is chosen as $[1.5,1.5,1.5,1.5]$. The Laplacian $L$ and the matrix $H$ are as follows:

$$
\begin{gathered}
L=\left[\begin{array}{llllllllll}
2 & -1 & 0 & 0 & -1 & 0 & 0 & 0 & 0 & 0 \\
-1 & 3 & -1 & -1 & 0 & 0 & 0 & 0 & 0 & 0 \\
0 & -1 & 2 & -1 & 0 & 0 & 0 & 0 & 0 & 0 \\
0 & -1 & -1 & 3 & -1 & 0 & 0 & 0 & 0 & 0 \\
-1 & 0 & 0 & -1 & 3 & -1 & 0 & 0 & 0 & 0 \\
0 & 0 & 0 & 0 & -1 & 3 & -1 & 0 & 0 & -1 \\
0 & 0 & 0 & 0 & 0 & -1 & 2 & 0 & -1 & 0 \\
0 & 0 & 0 & 0 & 0 & 0 & 0 & 1 & -1 & 0 \\
0 & 0 & 0 & 0 & 0 & 0 & -1 & -1 & 3 & -1 \\
0 & 0 & 0 & 0 & 0 & -1 & 0 & 0 & -1 & 2
\end{array}\right] \\
H
\end{gathered}
$$

The minimum eigenvalue of $(L+H)$ is 0.2569 . Here, we do not need to use the information of the interaction network topology. Since there are ten agents and one leader in the group, using exhaustive search, we can obtain the minimum eigenvalue of the possible spanning trees consisting ten agents and a leader. Therefore, we can choose $\gamma=0.1$ and $\theta=\lambda_{N}\left(L_{c}+H\right)=11$. For $\varepsilon=0.1, \varepsilon=0.01$ and $\varepsilon=0.001$, we obtain the following positive definite matrices:

$$
P(0.1)=\left[\begin{array}{llll}
1.9721 & 0.3313 & 1.3587 & 0.0498 \\
0.3313 & 6.5031 & 0.4756 & 1.9527 \\
1.3587 & 0.4756 & 3.4637 & 0.1310 \\
0.0498 & 1.9527 & 0.1310 & 1.3453
\end{array}\right],
$$




$$
P(0.01)=\left[\begin{array}{llll}
0.6184 & 0.0332 & 0.4266 & 0.0050 \\
0.0332 & 2.0461 & 0.0477 & 0.6177 \\
0.4266 & 0.0477 & 1.0879 & 0.0132 \\
0.0050 & 0.6177 & 0.0132 & 0.4261
\end{array}\right]
$$

and

$$
P(0.001)=\left[\begin{array}{llll}
0.1954 & 0.0033 & 0.1348 & 0.0005 \\
0.0033 & 0.6467 & 0.0048 & 0.1954 \\
0.1348 & 0.0048 & 0.3438 & 0.0013 \\
0.0005 & 0.1954 & 0.0013 & 0.1348
\end{array}\right],
$$

which all satisfy condition (3). Therefore, we obtain $\lambda_{\max }(P(\varepsilon)=0.1)=7.2757, \lambda_{\min }(P(\varepsilon)=$ $0.1)=0.6859, \lambda_{\max }(P(\varepsilon)=0.01)=2.2586, \lambda_{\min }(P(\varepsilon)=0.01)=0.2173, \lambda_{\max }(P(\varepsilon)=0.1)=$ 0.7129 and $\lambda_{\min }(P(\varepsilon)=0.1)=0.0687$. From the boundary of the initial states and the value of $P(\varepsilon)$, we can choose $c=4431.1, c=1309.2$ and $c=405.1444$ for $\varepsilon=0.1, \varepsilon=0.01$ and $\varepsilon=0.001$, respectively.

If we choose the constants $b=0$ and $D_{0}=0$, then we obtain $\rho=0.0560, \rho=0.5487$ and $\rho=5.4757$ for $\varepsilon=0.1, \varepsilon=0.01$ and $\varepsilon=0.001$, respectively. Fig. 1 shows the robust consensus tracking results of the ten agents under control protocol (4) to follow the leader. Figs. 1(a) and 1(b) plot the consensus tracking between the ten agents and the leader and the control signals of the ten agents, respectively, when $\varepsilon=0.1$. Figs. 1(c) and 1(d) plot the consensus tracking between the ten agents and the leader and the control signals of the ten agents, respectively, when $\varepsilon=0.01$. Figs. 1(e) and 1(f) plot the consensus tracking between the ten agents and the leader and the control signals of the ten agents, respectively, when $\varepsilon=0.001$.

Starting from the same initial states, Fig. 2 and Fig. 3 present the settling time of the system as a function of $\rho$ and $\varepsilon$, when $b=0, D_{0}=0$ for $\varepsilon=0.01$ and $\rho=0.056$, respectively. The figures show that, the smaller the value of $\rho$ or $\varepsilon$, the larger the settling time.

If we choose $b=0.5$ and $D_{0}=0$, after calculation, then we obtain $\rho=8.8665, \rho=93.1623$ and $\rho=950.1271$ for $\varepsilon=0.1, \varepsilon=0.01$ and $\varepsilon=0.001$, respectively. Fig. 4 shows the robust swarm tracking of the ten agents under control protocol (4) to follow the leader. Figs. 4(a) and 4(b) plot the evolution of the state difference between the ten agents and the leader and the control signals of the ten agents, respectively, when $\varepsilon=0.1$. Figs. 4(c) and 4(d) plot the evolution of the state difference between the ten agents and the leader and the control signals 
of the ten agents, respectively, when $\varepsilon=0.01$. Figs. 4(e) and 4(f) plot the evolution of the state difference between the ten agents and the leader and the control signals of the ten agents, respectively, when $\varepsilon=0.001$. It is obvious from Fig. 4 that the control protocol (4) is capable of achieving stable swarm tracking.

\section{Conclusions}

In this paper, we have investigated the robust semi-global consensus tracking and swarm tracking of multi-agent systems subject to actuator magnitude saturation and dead zone characteristic and input additive uncertainties and disturbances. We have designed tracking algorithms via a low-and-high gain feedback approach to deal with the two problems. Under the assumption that the system is asymptotically null controllable with bounded controls and the network is connected, robust semi-global consensus tracking of the multi-agent system is always reached when the known nonnegative constant in the uncertain element is zero and the saturation function has no dead zone characteristic; otherwise, robust semi-global swarm tracking can be attained. They both start from a given bounded set of initial conditions. Future work will be to investigate the proposed algorithms on directed and switching networks.

\section{REFERENCES}

[1] C.W. Reynolds, "Flocks, herds, and schools: A distributed behavioral model," Computer Graphics, ACM SIGGRAPH 87 Conference Proceedings, vol. 21, no. 4, pp. 25-34, 1987.

[2] T. Vicsek, A. Czirók, E. Ben-Jacob, O. Cohen and I. Shochet, "Novel type of phase transition in a system of self-driven particles," Physical Review Letters, vol. 75, no. 6, pp. 1226-1229, 1995.

[3] W. Ren, R. Beard and E. Atkins, "Information consensus in multivehicle cooperative control: collective group behavior through local interaction,” IEEE Control Systems Magazine, vol. 27, no. 2, pp. 71-82, 2007.

[4] H. Su and X. Wang, Pinning control of complex networked systems: Synchronization, consensus and flocking of networked systems via pinning, Springer, Heidelberg, 2013.

[5] X. Wang and G. Chen, "Synchronization in scale-free dynamical networks: robustness and fragility," IEEE Transactions on Circuits and Systems-I: Regular Papers, vol. 49, no. 1, pp. 54-62, 2002.

[6] W. He, F. Qian, Q. Han and J. Cao, "Lag quasi-synchronization of coupled delayed systems with parameter mismatch," IEEE Transactions on Circuits and Systems-I: Regular Papers, vol. 58, no. 6, pp. 1345-1357, 2011.

[7] J. Yu and L. Wang, "Group consensus in multi-agent systems with switching topologies and communication delays," Systems \& Control Letters, vol. 59, no. 6, pp. 340-348, 2010.

[8] B. Liu, T. Chu, L. Wang and G. Xie, "Controllability of a leaderCfollower dynamic network with switching topology," IEEE Transactions on Automatic Control, vol. 53, no. 4, pp. 1009-1013, 2008. 
[9] I. Belykh, V. Belykh and M. Hasler, "Blinking model and synchronization in small-world networks with a time-varying coupling," Physica D, vol. 195, no. 1-2, pp. 188-206, 2004.

[10] M. Porfiri, D. J. Stilwell, E. M. Bollt and J. D. Skufca, "Random talk: random walk and synchronizability in a moving neighborhood network," Physica D, vol. 224, no. 1-2, pp. 102-113, 2006.

[11] M. Porfiri and R. Pigliacampo, "Master-slave global stochastic synchronization of chaotic oscillators," SIAM Journal on Applied Dynamical Systems, vol. 7, no. 3, pp. 825-842, 2008.

[12] L. Wang, H. Dai, X. Kong and Y. Sun, "Synchronization of uncertain complex dynamical networks via adaptive control," Internatonal Journal of Robust and Nonlinear Control, vol. 19, no. 5, pp. 495-511, 2009.

[13] Y. Cao and W. Ren, "Distributed coordinated tracking with reduced interaction via a variable structure approach," IEEE Transactions on Automatic Control, vol. 57, no. 1, pp. 33-48, 2012.

[14] Y. Hong, J. Hu and L. Gao, "Tracking control for multi-agent consensus with an active leader and variable topology," Automatica, vol. 42, no. 7, pp. 1177-1182, 2006.

[15] W. Ren, "Multi-vehicle consensus with a time-varying reference state," Systems \& Control Letters, vol. 56, no. 7-8, pp. 474-483, 2007.

[16] K. Peng and Y. Yang, "Leader-following consensus problem with a varying-velocity leader and time-varying delays," Physica A, vol. 388, no. 2-3, pp. 193-208, 2009.

[17] H. Shi, L. Wang and T.G. Chu, "Virtual leader approach to coordinated control of multiple mobile agents with asymmetric interactions," Physica D, Vol. 213, pp. 51-65, 2006.

[18] J. Yao, R. Ordonez and V. Gazi, "Swarm tracking using artificial potentials and sliding mode control," Journal of Dynamic Systems, Measurement, and Control, vol. 129, no. 5, pp. 749-754, 2007.

[19] G. Xie and L. Wang, "Consensus control for a class of networks of dynamic agents," Internatonal Journal of Robust and Nonlinear Control, vol. 17, no. 10-11, pp. 941-959, 2007.

[20] W. Ren, "On consensus algorithms for double-integrator dynamics," IEEE Transactions on Automatic Control, vol. 53, no. 6, pp. 1503-1509, 2008.

[21] H. Su, X. Wang and Z. Lin, "Flocking of multi-agents with a virtual leader," IEEE Transactions on Automatic Control, vol. 54, no. 2, pp. 293-307, 2009.

[22] H. Su, X. Wang and G. Chen, "Rendezvous of multiple mobile agents with preserved network connectivity," Systems \& Control Letters, vol. 59, no. 5, pp. 313-322, 2010.

[23] Z. Li, Z. Duan, G. Chen and L. Huang, "Consensus of multiagent systems and synchronization of complex networks: A unified viewpoint," IEEE Transactions on Circuits and Systems-I: Regular Papers, vol. 57, no. 1, pp. 213-224, 2010.

[24] K. You and L. Xie, "Coordination of discrete-time multi-agent systems via relative output feedback," International Journal of Robust and Nonlinear Control, vol. 21, no. 13, pp. 1587-1605, 2011.

[25] K. You and L. Xie, "Network topology and communication data rate for consensusability of discrete-time multi-agent systems," IEEE Transactions on Automatic Control, vol. 56, no. 10, pp. 2262-2275, 2011.

[26] T. Li, M. Fu, L. Xie and J. Zhang, "Distributed consensus with limited communication data rate," IEEE Transactions on Automatic Control, vol. 56, no. 2, pp. 279-292, 2011.

[27] W. Yu, G. Chen, M. Cao and J. Kurths, "Second-order consensus for multi-agent systems with directed topologies and nonlinear dynamics," IEEE Transactions on Systems, Man and Cybernetics-Part B, vol. 40, no. 3, pp. 881-891, 2010.

[28] H. Su, G. Chen, X. Wang and Z. Lin, "Adaptive second-order consensus of networked mobile agents with nonlinear dynamics," Automatica, vol. 47, no. 2, pp. 368-375, 2011. 
[29] L. Cheng, Z. Hou, M. Tan, Y. Lin and W. Zhang, "Neural-network based adaptive leader-following control for multi-agent systems with uncertainties," IEEE Transactions on Neural Networks, vol. 21, no. 8, pp. 1351-1358, 2010.

[30] H. Wang and Y. Xie, "Prediction error based adaptive Jacobian tracking of robots with uncertain kinematics and dynamics," IEEE Transactions on Automatic Control, vol. 54, no. 12, pp. 2889-2894, 2009.

[31] Y. Li, J. Xiang and W. Wei, "Consensus problems for linear time-invariant multi-agent systems with saturation constraints," IET Control Theory and Applications, vol. 5, no. 6, pp. 823-829, 2011.

[32] J. Cortés, "Finite-time convergent gradient flows with applications to network consensus," Automatica, vol. 42, no. 11, pp. 1993-2000, 2006.

[33] Z. Liu and Z. Chen, "Discarded consensus of network of agents with state constraint," IEEE Transactions on Automatic Control, vol. 57, no. 11, pp. 2869-2874, 2012.

[34] Y. Zheng, Y. Zhu and L. Wang, "Consensus of heterogeneous multi-agent systems," IET Control Theory \& Applications, vol. 5, no. 16, pp. 1881-1888, 2011.

[35] Y. Zheng and L. Wang, "Consensus of heterogeneous multi-agent systems without velocity measurements," International Journal of Control, vol. 85, no. 7, pp. 906-914, 2012.

[36] A. Nedić, A. Ozdaglar and P. A. Parrilo, "Constrained consensus and optimization in multi-agent networks," IEEE Transactions on Automatic Control, vol. 55, no. 4, pp. 922-938, 2010.

[37] A. Abdessameud and A. Tayebi, "On consensus algorithms for double-integrator dynamics without velocity measurement and with input constraints," Systems \& Control Letters, vol. 59, no. 12, pp. 812-821, 2010.

[38] H. Su, M. Z. Q. Chen, J. Lam and Z. Lin, "Semi-global leader-following consensus of linear multi-agent systems with input saturation via low gain feedback," IEEE Transactions on Circuits and Systems-I: Regular Papers, vol. 60, no. 7, pp. 1881-1889, 2013.

[39] Z. Lin, Low Gain Feedback, Lecture Notes in Control and Information Sciences, vol. 240, Springer, London, 1998.

[40] C. Godsil and G. Royle, Algebraic Graph Theory, Graduate Texts in Mathematics, vol. 207, Springer, New York, 2001. 


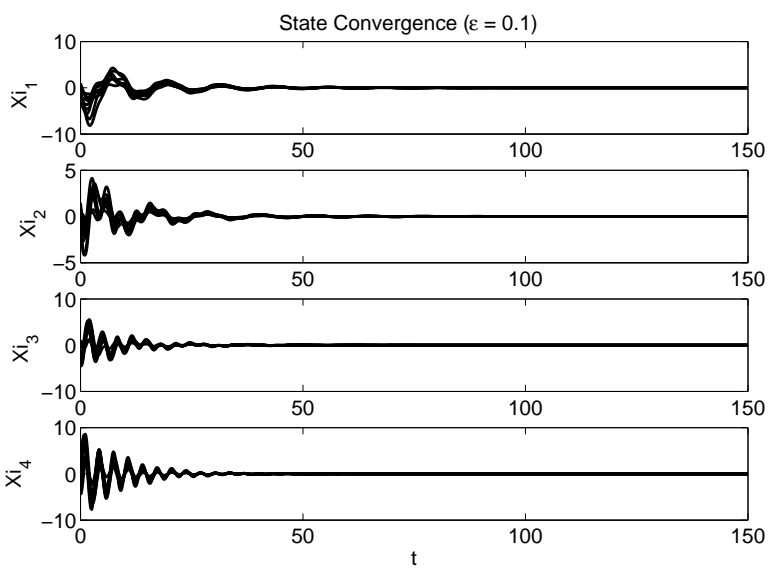

(a)

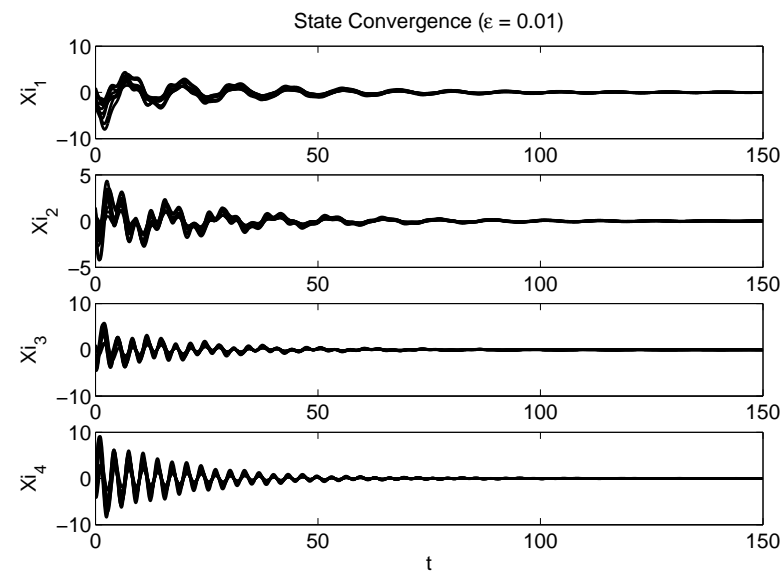

(c)
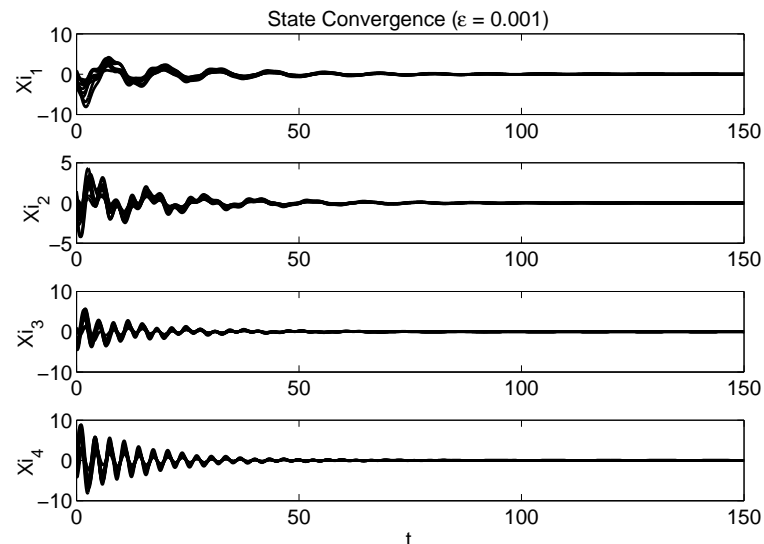

(e)

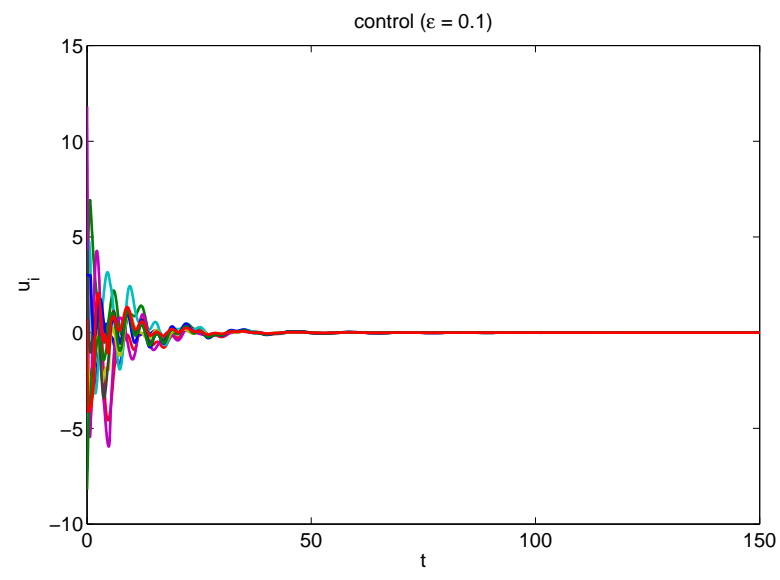

(b)

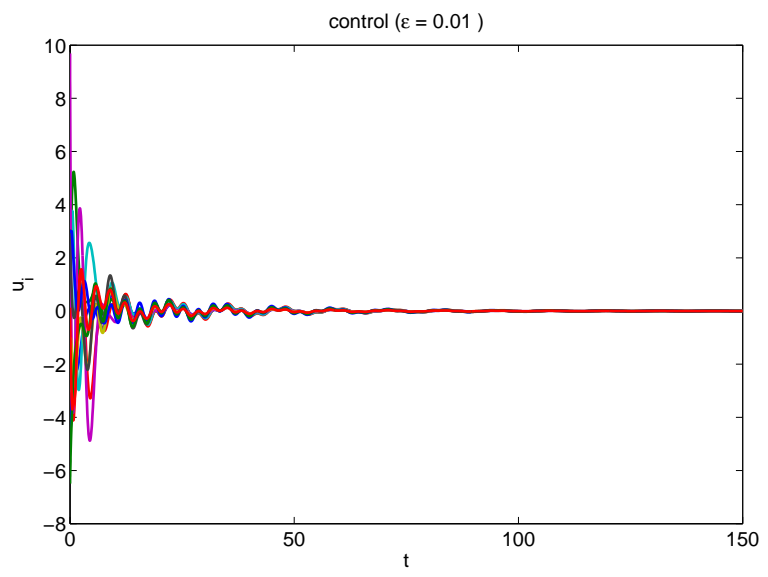

(d)

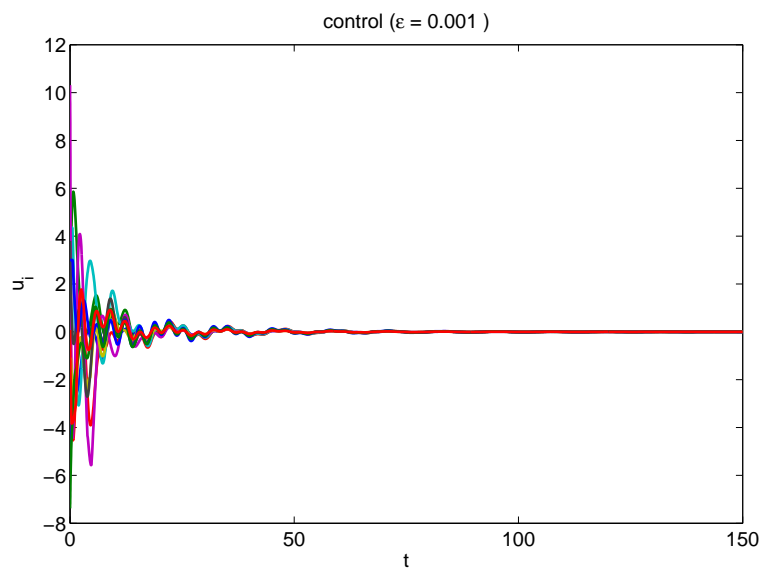

(f)

Fig. 1. Consensus tracking of ten agents with one leader under control protocol (4). 


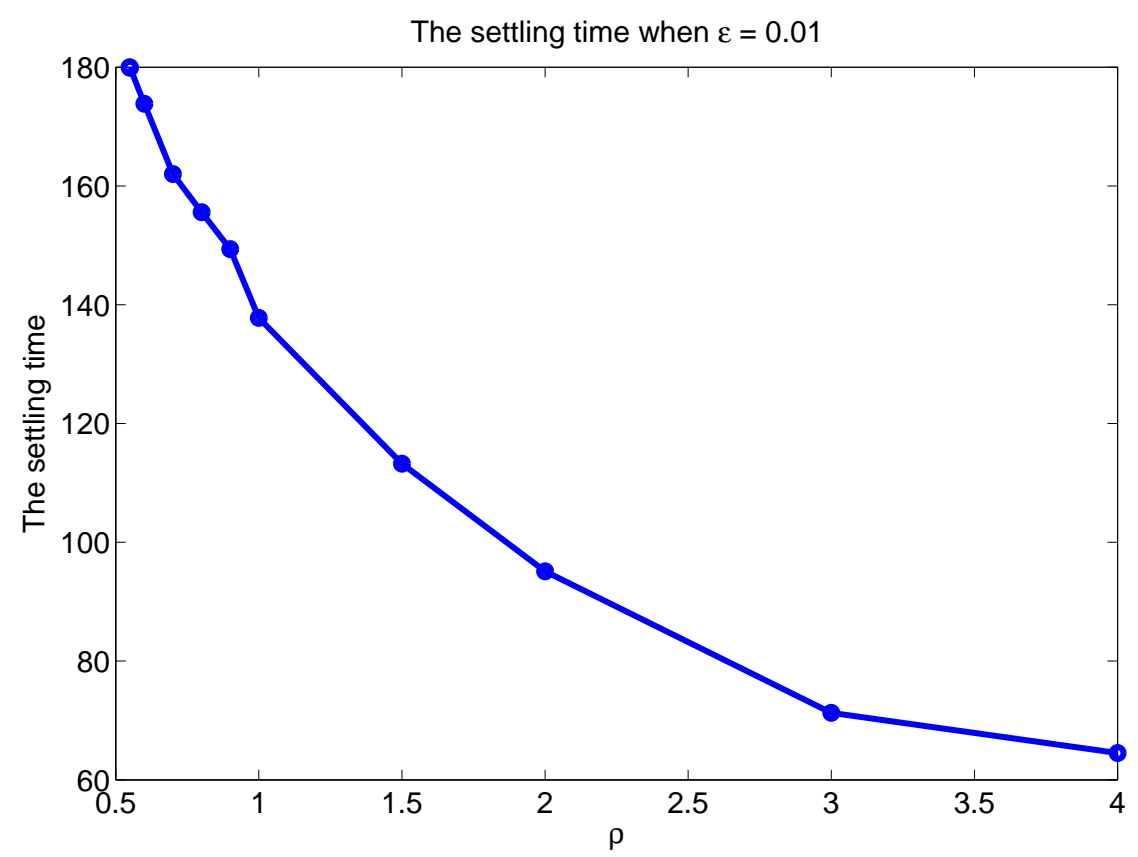

Fig. 2. The settling time of the system as a function of $\rho$, where the percentage set for the settling time of the system is chosen as $c_{t s}=0.04$.

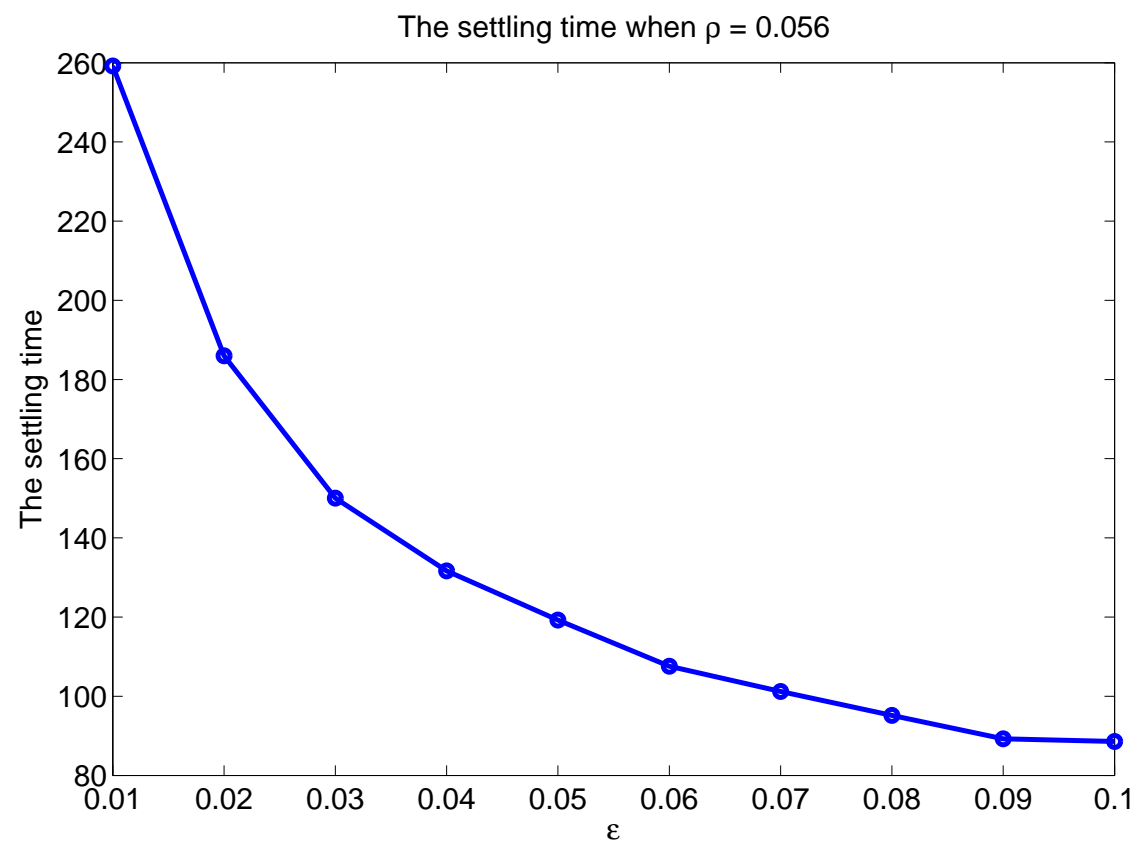

Fig. 3. The settling time of the system as a function of $\epsilon$, where the percentage set for the settling time of the system is chosen as $c_{t s}=0.04$. 

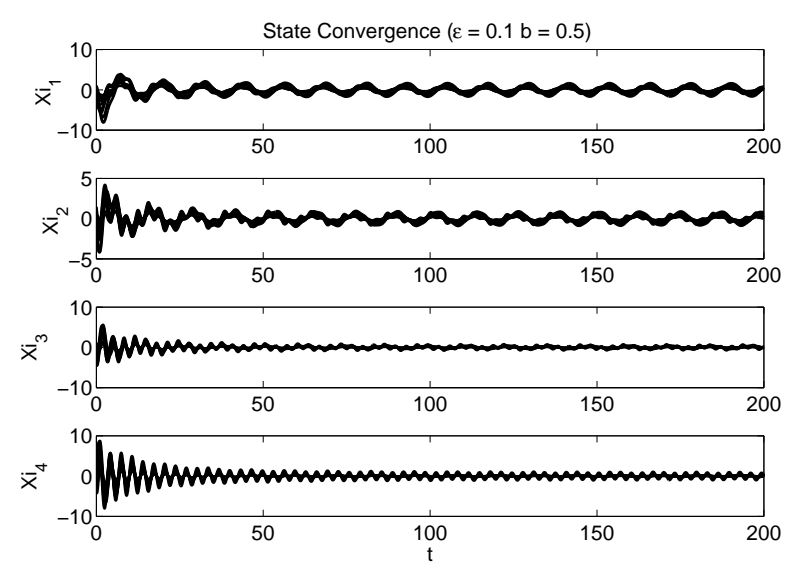

(a)

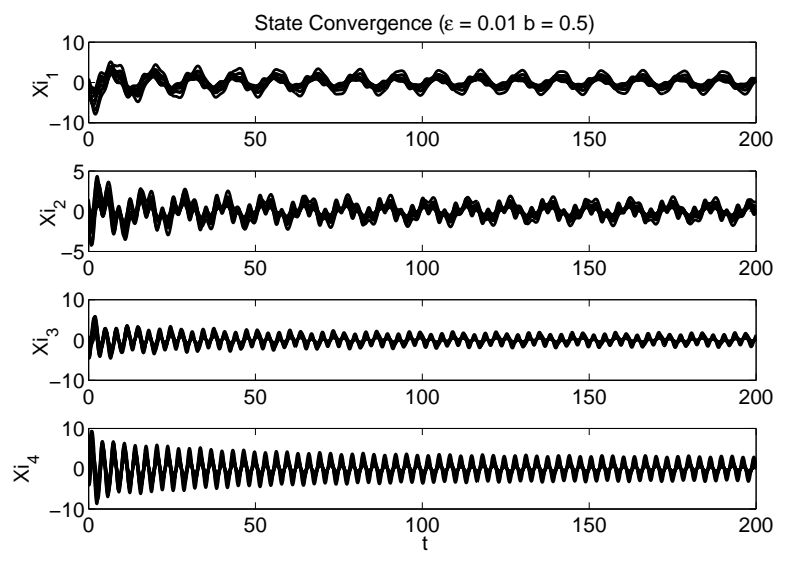

(c)
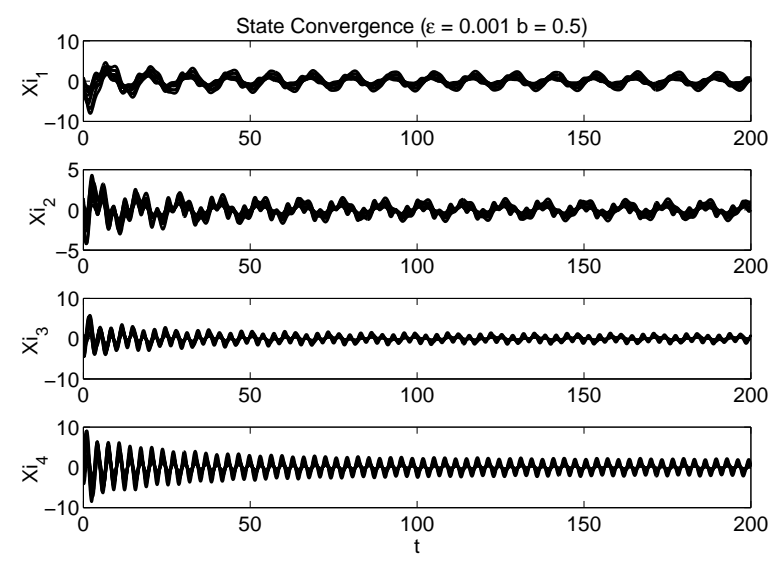

(e)

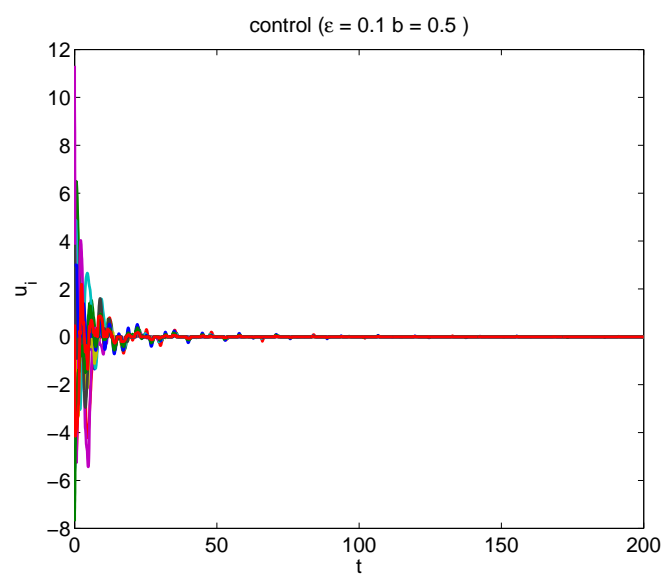

(b)

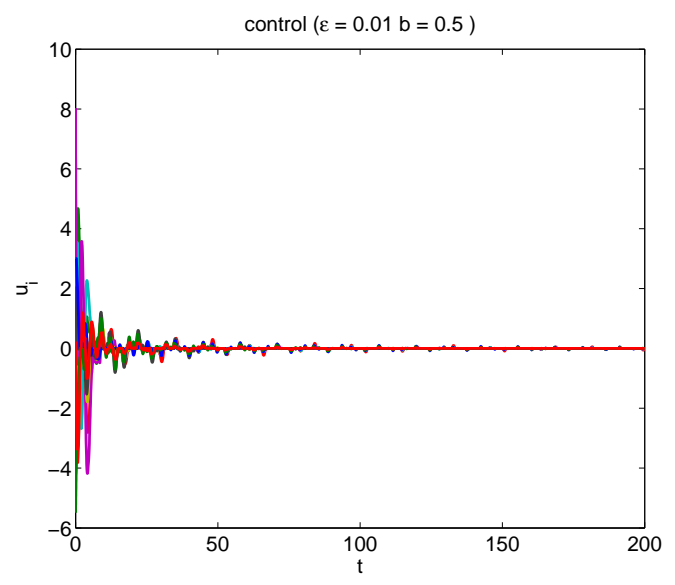

(d)

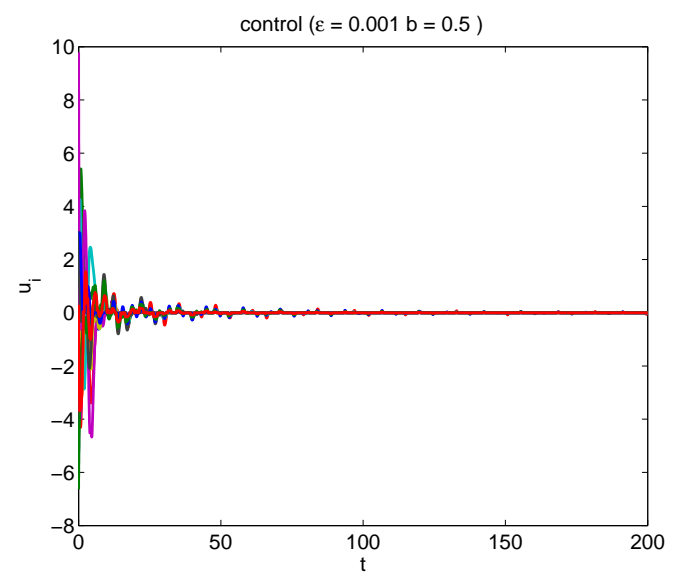

(f)

Fig. 4. Swarm tracking of ten agents with one leader under control protocol (4). 